\title{
A ressonância magnética e a artrorressonância magnética na lesão labral e condral do quadril: comparação com achados na artroscopia*
}

\author{
Magnetic resonance imaging and magnetic resonance \\ arthrography in labral and chondral lesion of the \\ hip: comparison with arthroscopy findings
}

\begin{abstract}
Marcos Emilio Kuschnaroff Contreras ${ }^{1}$, Sérgio Marcondes Brincas ${ }^{2}$, Ademar José de Oliveira PaEs JúnioR ${ }^{3}$, Getúlio Rodrigues de Oliveira Filho ${ }^{4}$, Francisco José BerRal de La Rosa ${ }^{5}$
\end{abstract}

\section{RESUMO}

Objetivo: Estudar a sensibilidade da ressonância magnética (RM) e da artrorressonância magnética (artro RM) para o diagnóstico das lesões do lábio acetabular e das lesões de cartilagem do quadril. Métodos: Foram incluídos 90 pacientes (90 quadris), submetidos à videoartroscopia do quadril, no período entre maio de 2004 e julho de 2007, na Ultra-

* Trabalho realizado no Ultralitho Centro Médico - Florianópolis (SC), Brasil, no Hospital Governador Celso Ramos - Florianópolis (SC), Brasil e na Clínica Imagem - Florianópolis (SC), Brasil.

1. Mestre, Chefe do Grupo de Quadril do Serviço de Ortopedia e Traumatologia do Hospital Governador Celso Ramos - Florianópolis (SC), Brasil.

2. Médico Radiologista da Clínica Imagem - Florianópolis (SC), Brasil.

3. Doutor, Médico Radiologista da Clínica Imagem - Florianópolis (SC), Brasil.

4. Doutor, Anestesiologista do Hospital Governador Celso Ramos - Florianópolis (SC), Brasil.

5. Doutor em Medicina, Chefe do Departamento de Alto Rendimento Esportivo - Universidade Pablo de Olavide-Sevilha - Espanha.

Endereço para correspondência: Marcos Emilio Kuschnaroff Contreras, Rua Abílio Costa 220, Córrego Grande - 88037-150 - Florianópolis, SC. Fax: (48) 3228-2031.

E-mail: dr.marcoscontreras@gmail.com

Recebido em 12/2/08. Aprovado para publicação em 30/5/08.

Copyright RBO2008 litho Centro Médico e no Hospital Governador Celso Ramos, ambos em Florianópolis, Santa Catarina. Dos 90 pacientes, $25(27,7 \%)$ realizaram RM, sem contraste, $54(60 \%)$ realizaram artro RM e 11 $(12,22 \%)$ realizaram os dois exames. Nos pacientes submetidos a artro RM, foi introduzido junto com o contraste um anestésico local (bupivacaína 0,5\%, ou ropivacaína $1 \%$, sem adrenalina). Em todos os casos, verificou-se a presença de lesão do lábio acetabular e de lesão condral durante a videoartroscopia. Resultados: Os autores encontraram maior sensibilidade para o diagnostico de lesão do lábio acetabular com a artro RM $(96,9 \%)$, do que com RM $(86,1 \%)$, sendo esta diferença estatisticamente significante. Quanto ao diagnóstico de lesão da cartilagem do quadril, a sensibilidade da artro RM foi

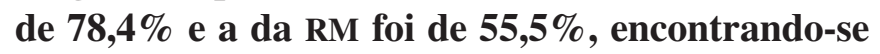
também diferença estatisticamente significante. Houve maior percentagem de falso-negativo na RM, tanto para o diagnostico de lesão labral quanto condral. Não foi encontrado nenhum caso de falso-positivo nesta série. A introdução de anestésico local para a realização de artro RM mostrou alivio da dor em 56,9\% dos casos. Conclusão: Os autores concluíram que a artro RM apresentou maior sensibilidade de diagnóstico que a RM para lesões do lábio acetabular e da cartilagem do quadril. A indiferen- 
ça ou a piora da dor, após a introdução de anestésico local na artro RM, não foi considerada como fator preditivo para lesões intra-articulares.

Descritores - Imagem por ressonância magnética; Artroscopia; Articulação do quadril

\section{ABSTRACT}

Objective: To study the sensitivity of magnetic resonance imaging (MRI) and magnetic resonance arthrography (MRA) for the diagnosis of acetabular labrum lesions and of hip cartilage lesions. Methods: 90 patients (90 hips) were included, who had been submitted to hip videoarthroscopy between May 2004 and July 2007, at the Ultralitho Medical Center and at the Governador Celso Ramos Hospital, both in the city of Florianópolis, State of Santa Catarina. Of the 90 patients, $25(27.7 \%)$ were submitted to MRI without contrast, 54 (60\%) were submitted to MRA, and 11 (12.22\%) were submitted to both MRI and MRA. In patients submitted to MRA, the contrast was given together with a local anesthetic (bupivacaine 0.5\%, or ropivacaine 1\%, without adrenaline). Acetabular labrum lesion and chondral lesion were seen under videoarthroscopy in all cases. Results: The authors found higher sensitivity to diagnose acetabular labrum lesion with MRA (96.9\%) than with MRI (86.1\%), such difference being statistically significant. With respect to the diagnosis of lesion in the hip cartilage, RMA sensitivity was $78.4 \%$, and MRI sensitivity was $55.5 \%$, which is also a statistically significant difference. There was a higher percent of false negative results in MRI, in the diagnosis of both labral lesion and chondral lesion. No false positive was found in this series. The introduction of a local anesthetic to perform MRA showed pain relief in $56.9 \%$ of the cases. Conclusion: The authors conclude that MRA presented higher diagnostic sensitivity than MRI for acetabular labrum lesions and hip cartilage lesions. Lack of difference in pain or the worsening of pain after introduction of a local anesthetic for MRA was not considered a predictive factor for intra-articular lesions.

Keywords - Magnetic resonance imaging; Arthroscopy; Hip joint

\section{INTRODUÇÃO}

O reconhecimento de lesões intra-articulares do quadril, como motivo de dor e de futuras alterações de caráter degenerativo, tem levado os pesquisadores à procura de métodos diagnósticos de maior sensibilidade o mais precoce possível ${ }^{(1-9)}$.

A introdução da videoartroscopia do quadril acrescentou mais uma ferramenta para o diagnóstico e o tratamento dessas lesões ${ }^{(10)}$. A indicação desta cirurgia deve ser criteriosa e baseada na anamnese, exame físico e comprovada por exames de imagem.

$\mathrm{O}$ presente estudo tem como objetivo comparar as lesões identificadas durante a videoartroscopia do quadril com as imagens visualizadas em ressonâncias magnéticas e artrorressonâncias previamente realizadas, como propósito de avaliar a sensibilidade de cada um destes dois exames.

\section{MÉTODOS}

No período entre maio de 2004 e julho de 2007, foram realizadas 90 videoartroscopias de quadril, consecutivas, com diagnóstico de lesão do lábio acetabular e/ ou condropatia coxofemoral, na Ultralitho Centro Médico e no Hospital Governador Celso Ramos, ambos em Florianópolis, Santa Catarina.

Os pacientes receberam indicação de realização de ressonância magnética e videoartroscopia do quadril por suspeita clínica de doença intra-articular do quadril, com base na anamnese e no exame físico.

Dos 90 pacientes, 25 (27,7\%) realizaram RM, sem contraste, enquanto $54(60 \%)$ realizaram artro RM e 11 pacientes $(12,22 \%)$ realizaram os dois exames.

Todos os exames foram feitos por meio de equipamentos MRI Giroscan T5 0,5T ${ }^{\circledR}$ (Phillips, Holanda) e MRI Eclipse 1,5T ${ }^{\circledR}$ (Picker, EUA), utilizando bobinas flexíveis dedicadas à articulação.

O protocolo de exame de RM e artro RM incluíram sequiências spin-eco axiais e coronais ponderadas em T1, com parâmetros TR/TE de 500-625/12-15, assim como seqüências axiais, coronais e sagitais ponderadas em DP (densidade de prótons) com saturação de gordura e STIR (inversion recovery), com parâmetros TR/TE de 2500-3500/40-45. A matriz utilizada foi 
de 256 x 256, com campo de visão (fov) entre 23 e 30 $\mathrm{cm}$. Número de excitações (nex) de 1 ou 2.

Nos exames de artro RM, foi realizado preparo com protocolo de injeção intra-articular de solução composta por $0,02 \mathrm{ml}$ de contraste paramagnético (gadolínio), 4,0ml de contraste iodado não-iônico e 3,0ml de bupivacaína $0,5 \%$, sem vasoconstritor, diluídos em $10,0 \mathrm{ml}$ de soro fisiológico $0,9 \%$. Em todos os casos o acesso foi realizado por via anterior, no nível do colo do fêmur, com agulha fina (22G), monitorado por radioscopia.

O objetivo da utilização do anestésico intra-articular é de realizar teste terapêutico, para avaliar a resposta do paciente quanto à dor no quadril após os exames. Foi questionado se houve melhora, piora ou indiferença da dor no quadril.

Todos os 90 pacientes foram submetidos a tratamento cirúrgico por videoartroscopia e todos os casos foram documentados. Dos pacientes, $41(45,5 \%)$ eram do sexo masculino e $49(54,4 \%)$ eram do sexo feminino, estando $51(56,6 \%)$ com o lado direito acometido e 41 $(45,5 \%)$ com o lado esquerdo. Em dois casos a sintomatologia era bilateral. A idade variou entre 17 e 58 anos, com média de 37 anos.

Os resultados foram analisados separadamente para RM e artro RM do quadril e comparados com os achados cirúrgicos.

Todos os exames de imagem foram avaliados pelo mesmo radiologista, assim como todos os procedimentos cirúrgicos foram realizados pelo mesmo cirurgião.

Para análise estatística, utilizou-se estatística descritiva e o teste $\mathrm{z}$ para proporções.

\section{RESULTADOS}

A RM do quadril, realizada em 36 pacientes, mostrou lesão do lábio acetabular em 31 casos $(86,1 \%)$, associada a condropatia acetabular em $19(52,7 \%)$ e foi negativa para o diagnóstico de condropatia em 12 casos $(33,3 \%)$. Em um caso o diagnóstico foi de lesão condral sem lesão labral e em dois casos o diagnóstico foi de avulsão da inserção na crista ilíaca ântero-inferior do músculo reto femoral, sem lesão labral ou condral associada. Em dois casos o exame foi normal, totalizando quatro casos sem diagnostico de lesão labral ou condral (tabela 1).

\section{TABELA 1}

Freqüência dos resultados da ressonância magnética do quadril

\begin{tabular}{lcc}
\hline \multirow{2}{*}{ Diagnóstico } & \multicolumn{2}{c}{ Freqüência } \\
\cline { 2 - 3 } & Absoluta & Relativa \\
\hline Lesão labral pura & 12 & $33,3 \%$ \\
Lesão labral com condropatia & 19 & $52,7 \%$ \\
Lesão condral pura & 1 & $2,7 \%$ \\
Ausência de lesão labral e condral & 4 & $11,1 \%$ \\
Total & 36 & $100 \%$ \\
\hline
\end{tabular}

Nos casos em que foi realizada a RM, a videoartroscopia do quadril revelou lesão do lábio acetabular e condropatia acetabular e/ou femoral, em diferentes graus de lesão, em todos os 36 casos (100\%). Assim, a RM não detectou lesão labral em cinco casos $(13,8 \%)$ e lesão condral em 16 casos (44,4\%). O diagnóstico de lesão labral por imagem foi confirmado pela videoartroscopia em 31 casos $(86,1 \%)$, enquanto o diagnóstico da lesão condral foi confirmado em 20 casos $(55,5 \%)$. Não foi encontrado nenhum caso com falsopositivo para lesões labrais, tampouco para lesões da cartilagem (tabela 2).

A artro RM do quadril foi realizada em 65 pacientes e evidenciou lesão pura do lábio acetabular em 12 casos $(28,5 \%)$ e lesão labral associada a lesão condral

TABELA 2

Comparação entre as lesões detectadas na ressonância magnética e na videoartroscopia do quadril

\begin{tabular}{|c|c|c|c|c|c|c|}
\hline & $\begin{array}{c}\text { № de lesões } \\
\text { RM }\end{array}$ & $\begin{array}{l}\text { № de lesões } \\
\text { Artroscopia }\end{array}$ & $\begin{array}{c}\text { Falso- } \\
\text { (\%) }\end{array}$ & $\begin{array}{c}\text { Falso+ } \\
\text { (\%) }\end{array}$ & $\begin{array}{c}\text { Sensibilidade } \\
(\%)\end{array}$ & $\begin{array}{c}\text { Especificidade } \\
(\%)\end{array}$ \\
\hline Lábio & 31 & 36 & 13,8 & 0,0 & 86,1 & - \\
\hline Cartilagem & 20 & 36 & 44,4 & 0,0 & 55,5 & - \\
\hline
\end{tabular}

№ = número Falso- = falso-negativo Falso+ = falso-positivo 
em $29(69,0 \%)$. Apenas em um caso (2,2\%) foi identificada ausência de lesão labral ou condral, evidenciando apenas um cisto subcortical anterior conhecido como "herniation pitt" (tabela 3).

TABELA 3

Freqüência dos resultados da artrorressonância magnética do quadril

\begin{tabular}{lcc}
\hline \multirow{2}{*}{ Diagnóstico } & \multicolumn{2}{c}{ Freqüência } \\
\cline { 2 - 3 } & Absoluta & Relativa \\
\hline Lesão labral pura & 12 & $18,4 \%$ \\
Lesão labral com condropatia & 51 & $78,4 \%$ \\
Ausência de lesão labral e condral & 2 & $3 \%$ \\
Total & 65 & $100 \%$ \\
\hline
\end{tabular}

Nos 65 casos em que foi realizada a artro RM, a videoartroscopia do quadril revelou lesão do lábio acetabular e condropatia acetabular e/ou femoral, em diferentes graus, em todos os casos (100\%). O diagnóstico correto da lesão do lábio acetabular pela artro RM ocorreu em 63 casos $(96,9 \%)$ e a lesão da cartilagem foi corretamente diagnosticada em 51 casos $(78,4 \%)$. O diagnostico de lesão labral não foi realizado em dois casos $(3 \%)$; houve falha no diagnostico por imagem da lesão condral em 14 casos $(21,5 \%)$. Não foi encontrado diagnóstico falso-positivo para lesão labral e condral na artro RM (tabela 4).
A tabela 5 mostra os percentuais de valores falsonegativos e de sensibilidade, comparativamente entre a RM e a artro RM, no diagnóstico de lesão labral e condral.

A tabela 6 mostra a comparação entre a sensibilidade do diagnóstico da lesão do lábio acetabular e das lesões condrais realizada pela RM e pela artro RM. Utilizou-se o teste $\mathrm{z}$ para proporções $(\alpha=0,05)$. O teste mostra diferença estatística significativa entre RM e artro RM para o diagnóstico de lesão do lábio acetabular, e das lesões de cartilagem utilizando-se estes métodos de imagem.

\section{TABELA 6}

Comparação entre a sensibilidade diagnóstica da ressonância magnética e artrorressonância magnética, utilizando-se o teste $z$ para proporções $(\alpha=0,05)$

\begin{tabular}{lccc}
\hline & RM & Artro RM & p valor \\
\hline Lábio & 0,861 & 0,969 & $0,02^{*}$ \\
Cartilagem & 0,555 & 0,784 & $0,008^{*}$ \\
Numero de casos & 36 & 65 &
\end{tabular}

* = valor com significância estatística

Dos 90 pacientes incluídos no estudo, 11 (12,2\%) realizaram os dois exames (RM e artro RM). Destes 11 pacientes, em três casos a RM convencional mostrou ausência de lesão labral ou condral, em quatro casos havia lesão labral pura, em um caso lesão condral pura

TABELA 4

Comparação entre as lesões detectadas na artrorressonância magnética e na videoartroscopia do quadril

\begin{tabular}{lcccccc}
\hline & $\begin{array}{c}\text { № de lesões } \\
\text { Artro RM }\end{array}$ & $\begin{array}{c}\text { No de lesões } \\
\text { Artroscopia }\end{array}$ & $\begin{array}{c}\text { Falso- } \\
(\%)\end{array}$ & $\begin{array}{c}\text { Falso+ } \\
(\%)\end{array}$ & $\begin{array}{c}\text { Sensibilidade } \\
\text { (\%) }\end{array}$ & $\begin{array}{c}\text { Especificidade } \\
\text { (\%) }\end{array}$ \\
\hline Lábio & 63 & 65 & 3 & 0,0 & 96,9 & - \\
Cartilagem & 51 & 65 & 21,5 & 0,0 & 78,4 & - \\
\hline
\end{tabular}

№ = número Falso- = falso-negativo $\mathrm{Falso}+=$ falso-positivo

TABELA 5

Comparação entre ressonância magnética e artrorressonância magnética

\begin{tabular}{lcccc}
\hline & $\begin{array}{c}\text { Falso-negativo (\%) } \\
\text { RM }\end{array}$ & $\begin{array}{c}\text { Falso-negativo (\%) } \\
\text { Artro RM }\end{array}$ & $\begin{array}{c}\text { Sensibilidade (\%) } \\
\text { RM }\end{array}$ & $\begin{array}{c}\text { Sensibilidade (\%) } \\
\text { Artro RM }\end{array}$ \\
\hline Lábio & 13,8 & 3 & 86,1 & 96,9 \\
Cartilagem & 44,4 & 21,5 & 55,5 & 78,4 \\
\hline
\end{tabular}


e em três havia lesão tanto da cartilagem quanto do lábio.

Apenas nos três casos em que havia lesão labral e condral, houve concordância com os achados na artro RM, e foram confirmados pela cirurgia.

Nos pacientes submetidos à artro RM, a resposta em relação à dor após a aplicação de anestésico intra-articular no quadril foi: redução da dor em 37 casos $(56,9 \%)$, indiferença em 20 casos $(30,7 \%)$ e piora em oito casos $(12,3 \%)$ (tabela 7$)$.

\section{TABELA 7}

Freqüências dos resultados obtidos com teste terapêutico de anestésico intra-articular

\begin{tabular}{lcc}
\hline \multirow{2}{*}{$\begin{array}{c}\text { Resultado do teste } \\
\text { terapêutico }\end{array}$} & \multicolumn{2}{c}{ Freqüência } \\
\cline { 2 - 3 } & Absoluta & Relativa \\
\hline Melhora & 37 & $56,9 \%$ \\
Indiferente & 20 & $30,7 \%$ \\
Piora & 8 & $12,3 \%$ \\
Total & 65 & $100 \%$ \\
\hline
\end{tabular}

Para a realização do teste terapêutico, utilizamos bupivacaína $0,5 \%$ sem vasoconstritor, $3 \mathrm{ml}$, em 36 casos, e ropivacaína $1 \%$ sem vasoconstritor, $3 \mathrm{ml}$ em 28 casos. O resultado deste teste está descrito na tabela 8 .

\section{TABELA 8}

Freqüências dos resultados obtidos com teste terapêutico de anestésico intra-articular, separados por droga

\begin{tabular}{|c|c|c|c|c|}
\hline \multirow{3}{*}{$\begin{array}{l}\text { Resultado } \\
\text { do teste } \\
\text { terapêutico }\end{array}$} & \multirow{2}{*}{\multicolumn{2}{|c|}{$\begin{array}{c}\text { Bupivacaína } 0,5 \% \\
\text { Freqüência }\end{array}$}} & \multirow{2}{*}{\multicolumn{2}{|c|}{$\begin{array}{c}\text { Ropivacaína } 1 \% \\
\text { Freqüência }\end{array}$}} \\
\hline & & & & \\
\hline & Absoluta & Relativa & Absoluta & Relativa \\
\hline Melhora & 22 & $61,1 \%$ & 15 & $53,7 \%$ \\
\hline Indiferente & 11 & $30,5 \%$ & 9 & $32,1 \%$ \\
\hline Piora & 4 & $11,1 \%$ & 4 & $14,8 \%$ \\
\hline Total & 36 & $100 \%$ & 28 & $100 \%$ \\
\hline
\end{tabular}

A tabela 9 mostra a diferença de sensibilidade ao teste terapêutico utilizando-se a bupivacaína $0,5 \%$ e a ropivacaína, através do teste z para proporções. Não houve diferença estatística significante no uso destas diferentes drogas.

\section{TABELA 9}

Comparação entre a sensibilidade ao teste terapêutico com o uso de bupivacaína e ropivacaína intra-articular, utilizando-se o teste $z$ para proporções $(\alpha=0,05)$

\begin{tabular}{lccc}
\hline & Bupivacaína & Ropivacaína & p valor \\
\hline Melhora & 0,611 & 0,537 & 0,26 \\
Indiferente & 0,305 & 0,321 & 0,43 \\
Piora & 0,111 & 0,148 & 0,35 \\
Numero de casos & 36 & 28 & \\
\hline
\end{tabular}

\section{DISCUSSÃO}

Edward et al registraram a falha do diagnostico de lesões condrais com a RM, principalmente aquelas menores que $1 \mathrm{~cm}$. Encontraram também casos de falso-positivo para lesões labrais quando a imagem mostrava lesões próximas ao ligamento transverso ${ }^{(1)}$.

Encontramos uma quantidade de exames com falso-negativo para a RM e artro RM semelhante aos descritos por Byrd et al e Keeney et al; contudo, não detectamos nenhum caso de falso-positivo. Não foi possível realizar o cálculo de especificidade, pois não identificamos nenhum caso de falso-positivo ${ }^{(2-3)}$.

Leunig et al avaliaram 23 pacientes com suspeita clinica de lesão labral, por meio da artro RM e da artroscopia. Obtiveram $63 \%$ e $71 \%$ para sensibilidade e especificidade nas lesões labrais, respectivamente. Descreveram um método de avaliação da localização da lesão utilizando a comparação com os ponteiros de um relógio ${ }^{(4)}$. No nosso estudo, não realizamos avaliações quanto à localização ou acurácia da lesão.

Czerny et al correlacionaram os achados cirúrgicos e anatômicos do complexo cápsulo-labral, com a artro RM. Realizaram o exame de imagem em 40 pacientes e seis espécimes de cadáveres. Todos os pacientes foram submetidos à artrotomia. Os autores mostraram que o lábio acetabular é uma estrutura triangular, não existe sulco sublabral, e tem intensidade de sinal baixa e homogênea na RM. Pode haver um recesso entre o lábio e a cápsula. A sensibilidade da artro RM para diagnóstico e estadiamento foi de $91 \%$, a especificidade foi de $71 \%$ e a acurácia, de $88 \%$. Concluíram que a artro RM apresenta excelente visualização do lábio normal e das patologias do complexo cápsulolabral $^{(5)}$. 
Czerny et al avaliaram a acurácia da RM e da artro RM para o diagnóstico de lesão do lábio acetabular. Dos pacientes, 40 foram submetidos à artroscopia do quadril, depois de realizadas RM e artro RM. O lábio foi avaliado na sua morfologia, intensidade do sinal, presença de lesão e fixação ao acetábulo. A sensibilidade da RM foi de $80 \%$ e da artro RM, de $95 \%$, enquanto a acurácia da RM foi de $65 \%$ e da artro RM foi de $88 \%$. Concluem que a artro RM é o melhor método para o diagnóstico de lesão labral ${ }^{(6)}$.

Em estudo semelhante realizado pelos mesmos autores, a sensibilidade da RM foi de apenas $35 \%$ e sua acurácia de $36 \%$, enquanto a sensibilidade da artro RM foi de $90 \%$ e a acurácia, de $91 \%{ }^{(7)}$. Isso pode denotar uma curva de aprendizado na leitura do exame sem contraste.

Mitchell et al correlacionaram achados da artro RM, ultra-sonografia, exame clínico e artroscopia do quadril. Estudaram 25 pacientes que foram submetidos à videoartroscopia do quadril. O exame clínico revelou que a limitação do movimento e a dor nos testes de flexão, abdução e rotação externa, comparados com o lado contra-lateral, foram positivos para lesões intraarticulares. Todas as radiografias convencionais foram normais. A artro RM foi realizada em 24 pacientes, com grande número de resultados negativos, o que não excluiu a possibilidade de lesão intra-articular. A artroscopia, nesses casos, revelou $100 \%$ de quadris com alguma lesão intra-articular ${ }^{(8)}$.

Leunig et al estudaram 14 pacientes com displasia acetabular submetidos a osteotomia periacetabular e 14 pacientes com impacto femoroacetabular. Realizaram radiografias convencionais e artro RM em todos os casos. Encontraram lesão do lábio acetabular em $64 \%$ dos casos em ambos os grupos, hipertrofia labral em $86 \%$ dos casos com displasia e em nenhum caso com impacto. Cistos paralabrais foram encontrados em $71 \%$ dos casos de displasia e em $21 \%$ dos casos com impacto femoroacetabular ${ }^{(9)}$.

Keeney et al realizaram artro RM, seguida de artroscopia do quadril, em 102 quadris consecutivos com suspeita clínica de lesão labral. A artro RM foi capaz de diagnosticar $76 \%$ das lesões labrais, com 4,9\% de falso-positivos. Quanto à lesão condral, o diagnóstico por imagem foi realizado em $62,7 \%$ dos casos. Os autores estudaram a sensibilidade, a especificidade, o valor preditivo positivo e negativo e a acurácia da artro RM para diagnostico das lesões labrais e condrais. Concluíram que, apesar de o exame apresentar valor preditivo positivo alto para essas lesões, tem sensibilidade limitada. O falso-negativo não exclui lesões intra-articulares ${ }^{(3)}$.

Byrd et al estudaram a confiança da avaliação clínica, da RM, artro RM e do teste terapêutico com bupivacaína intra-articular, para o diagnostico de lesões intra-articulares do quadril. Utilizaram a cirurgia artroscópica como diagnóstico definitivo. O exame clínico mostrou que havia lesão intra-articular com $98 \%$ de precisão, mas falhou na determinação da natureza da lesão. A RM mostrou $42 \%$ de falso-negativo e $10 \%$ de falso-positivo, enquanto a artro RM apresentou apenas $8 \%$ de falso-negativo; entretanto, mostrou o dobro de falso-positivo. O teste terapêutico elevou a confiança em $90 \%$, quanto ao diagnostico das lesões intra-articulares do quadril ${ }^{(2)}$.

Chan et al utilizaram a artro RM com imagens de reformatação radial com cortes sagitais verdadeiros, e concluíram que esta técnica aumenta o sucesso do diagnóstico das lesões labrais e ajuda a escolha de portais de artroscopia, aumentando a precisão das técnicas cirúrgicas $^{(10)}$.

Contrapondo-se à literatura, Mintz et al estudaram a eficácia da RM na detecção das lesões labrais e condrais. Estudo retrospectivo simples cego, com dois observadores, avaliou 92 pacientes submetidos à RM e depois à artroscopia. Os radiologistas observadores conseguiram identificar corretamente $95 \%$ das lesões labrais e de $86 \%$ a $92 \%$ das lesões condrais grau I, assim demonstrando que, com protocolo de observação adequado, não haveria necessidade do uso de contraste na RM, para o diagnóstico das lesões labrais e condrais do quadril ${ }^{(11)}$.

Amaral et al realizaram estudo comparativo entre os métodos de RM, artro RM e artrotomografia computadorizada (artro CT), em 20 pacientes, tendo como padrão ouro a videoartroscopia do quadril. Dois observadores avaliaram todos os exames. Obtiveram 93,7\% de sensibilidade no diagnostico de lesão do lá- 
bio acetabular com artro RM, e 75\% de sensibilidade no diagnóstico de lesão condral com artro CT, e assim consideraram estes como os melhores exames para o diagnostico das lesões labral e condral, respectivamente. Houve concordância interobservador estatisticamente significante. Os autores salientaram a importância do sulco labral póstero-superior como variante do normal, podendo ser confundido com lesão da base do lábio(*). Seus resultados são concordantes com os nossos e com a literatura sobre a lesão labral; contudo, existem poucos relatos do uso da artro CT, mesmo para o diagnóstico da lesão condral.

Schmid et al sustentaram que a artro RM tem baixa concordância interobservadores para o diagnóstico de lesões da cartilagem tanto do acetábulo, quanto do fêmur. Mostraram que a lesão condral mais freqüente é na região ântero-superior do acetábulo(12).

Toomayan et al estudaram a sensibilidade da RM e da artro RM na avaliação das lesões do lábio acetabular. Estudaram 51 quadris (48 pacientes) e realizaram RM com campo alargado $(30-38 \mathrm{~cm})$ em 14 quadris, e com campo reduzido $(14-20 \mathrm{~cm})$ em sete quadris. Realizaram artro RM com campo reduzido $(14-20 \mathrm{~cm}) \mathrm{em}$ 30 pacientes. Concluíram que a artro RM com campo reduzido foi mais sensível para o diagnóstico da lesão do lábio acetabular do que qualquer $\mathrm{RM}^{(13)}$.

Concordamos com Byrd et al, Keeney et al e Czerny et al, que mostraram que a sensibilidade da artro RM é maior que a da RM para o diagnóstico das lesões labrais e condrais do quadril ${ }^{(2-3,7)}$.

Byrd et al descreveram a relevância do uso de droga anestésica intra-articular, e que a melhora da dor elevaria o fator preditivo positivo ${ }^{(2)}$. Não obtivemos número de casos com melhora da dor com o uso de anestésico que fosse expressivo, mas nos chamou atenção o número de casos em que o uso foi indiferente. Acreditamos que havendo demora na elaboração do diagnóstico e, por sua vez, retarde na indicação do exame, talvez ocorra agravamento do processo inflamatório

* Amaral DT, Polesello G, Honda E, Lederman HM. Lesões condrais e labrais no quadril: comparação da RM, artro RM, artro CT e artroscopia. In: $38^{\circ}$ Congresso Brasileiro de Ortopedia e Traumatologia. Anais. Fortaleza, 2006. sinovial, o que levaria à diminuição do $\mathrm{pH}$ intra-articular, diminuindo a ação do anestésico local. Outra hipótese, para que a melhora da dor não ocorresse em número maior de casos seria o pouco volume de anestésico administrado. Portanto, acreditamos que a melhora da dor com a introdução do anestésico intra-articular pode ser considerada como confirmação de que a dor do paciente refere-se à lesão intra-articular do quadril; contudo, a ausência de melhora, ou piora da dor, não exclui a possibilidade de haver lesão intraarticular. Naturalmente, este dado deve ser confrontado com o exame de imagem. Estudos prospectivos randomizados com diferentes concentrações e diferentes anestésicos certamente ajudariam a resolver esta questão.

\section{CONCLUSÕES}

A artro RM tem sensibilidade superior à da RM, para o diagnóstico das lesões do lábio acetabular e da cartilagem da articulação coxo femoral.

A injeção de anestésico intra-articular na realização da artro RM mostrou-se eficaz no alívio da dor em 56,9\% dos casos, mas a ausência de melhora da dor ou piora da dor com sua aplicação não pode ser usada como fator preditivo de lesão intra-articular do quadril.

\section{REFERÊNCIAS}

1. Edwards DJ, Lomas D, Villar RN. Diagnosis of the painful hip by magnetic resonance imaging and arthroscopy. J Bone Joint Surg Br. 1995:77(3):374-6.

2. Byrd JW, Jones KS. Diagnostic accuracy of clinical assessment, magnetic resonance imaging, magnetic resonance arthrography, and intra-articular injection in hip arthroscopy patients.Am J Sports Med. 2004;32(7):1668-74.

3. Keeney JA, Peelle MW, Jackson J, Rubin D, Maloney WJ, Clohisy JC. Magnetic resonance arthrography versus arthroscopy in the evaluation of articular hip pathology. Clin Orthop Relat Res. 2004;(429):163-9.

4. Leunig M, Werlen S, Ungersbock A, Ito K, Ganz R. Evaluation of the acetabular labrum by MR arthrography. J Bone Joint Surg Br. 1997;79(2):230-4. Erratum in: J Bone Joint Surg Br. 1997;79(4):693.

5. Czerny C, Hofmann S, Urban M, Tschauner C, Neuhold A, Pretterklieber M, et al. MR arthrography of the adult acetabular capsular-labral complex: correlation with surgery and anatomy. AJR Am J Roentgenol. 1999;173(2):345-9. 
6. Czerny C, Kramer J, Neuhold A, Urban M, Tschauner C, Hofmann S. [Magnetic resonance imaging and magnetic resonance arthrography of the acetabular labrum: comparison with surgical findings]. Rofo. 2001;173(8):702-7. German

7. Czerny C, Hofmann S, Neuhold A, Tschauner C, Engel A, Recht MP, et al. Lesions of the acetabular labrum: accuracy of MR imaging and MR arthrography in detection and staging.Radiology. 1996;200(1):225-30.

8. Mitchell B, McCrory P, Brukner P, O’Donnell J, Colson E, Howells R. Hip joint pathology: clinical presentation and correlation between magnetic resonance arthrography, ultrasound, and arthroscopic findings in 25 consecutive cases. Clin J Sport Med. 2003;13(3):152-6.

9. Leunig M, Podeszwa D, Beck M, Werlen S, Ganz R. Magnetic Resonance Artrography of Labral Disorders in hip with displasia and impingement. Clin Orthop Relat Res. 2004;(418):74-80.
10. Chan YS, Lien LC, Hsu HL, Wan YL, Lee MS, Hsu KY, Shih $\mathrm{CH}$. Evaluating hip labral tears using magnetic resonance arthrography: a prospective study comparing hip arthroscopy and magnetic resonance arthrography diagnosis. Arthroscopy. 2005;21(10): 1250 .

11. Mintz DN, Hooper T, Connell D, Buly R, Padgett DE, Potter HG. Magnetic resonance imaging of the hip: detection of labral and condral abnormalities using noncontrast imaging. Arthroscopy. 2005;21(4):385-93.

12. Schmid MR, Notzli HP, Zanetti M, Wyss TF, Hodler J. Cartilage lesions in the hip: Diagnostic effectiveness of MR arthrography. Radiology. 2003;226(2):382-6.

13. Toomayan GA, Holman WR, Major NM, Kozlowicz SM, Vail TP. Sensitivity of MR Arthrography in the evaluation of acetabular labral tears. AJR Am J Roentgenol. 2006;186(2): 449-53.

Declaração de inexistência de conflitos de interesse: Declaramos para os devidos fins que não há qualquer conflito de interesse que impeça a publicação deste artigo. 